\title{
Carbon nanotube-zinc stannate nanohybrids as supercapacitor electrodes
}

\author{
A.K. Chakraborty ${ }^{1,2 *}$, N. Chakrabarty ${ }^{1,2}$ and A. Sarkar ${ }^{2,3}$ \\ ${ }^{1}$ Department of Physics, NIT Durgapur, Durgapur-713209, India \\ ${ }^{2}$ Centre of Excellence in Advanced Materials, NIT Durgapur, Durgapur-713209, India \\ ${ }^{3}$ Department of Metallurgical and Materials Engineering, NIT Durgapur, Durgapur-713209, India \\ *Email: amitkc61@gmail.com
}

Zinc stannate is a ternary oxide, which is known for its stable properties under extreme conditions and for its higher electron mobility when compared to its binary counterparts. The multifunctional material exists as two types of oxides with different $\mathrm{Zn} / \mathrm{Sn} / \mathrm{O}$ ratio and crystallographic structure: the orthorhombic $\mathrm{ZnSnO}_{3}$ (hereafter referred to as ZTO) and the cubic $\mathrm{Zn}_{2} \mathrm{SnO}_{4}$. ZTO is known to have high electrical conductivity, high electron mobility and low visible absorption, which makes it suitable for a wide range of applications such as photocatalysis [1], lithium ion batteries [2], dye-sensitized solar cells [3] etc. On the other hand, multiwall carbon nanotubes (MWCNTs) are amongst the most studied nanostructures for their many interesting properties suitable for various applications including supercapacitor electrodes [4]. However, the composites of CNT and ZTO have not received enough attention of the researchers.

In this work, we report the synthesis of a hybrid nanostructure composed of CNT and ZTO with the aim of developing new generation electrode material for supercapacitors. A simple hydrothermal method was adopted in our approach to synthesise the nanohybrid using $\left(\mathrm{Zn}\left(\mathrm{NO}_{3}\right)_{2}\right)$ and tin chloride $\left(\mathrm{SnCl}_{2}\right)$ as precursors to zinc and tin, respectively as this method offers good control over the composition and morphology of the material with high reproducibility. The microstructural and compositional analyses were carried out using powder x-ray diffraction (XRD), high-resolution transmission electron microscopy (HRTEM) and energy dispersive analysis by $\mathrm{x}$-rays (EDAX) and the electrochemical properties of the nanocomposite were evaluated by cyclic voltammetry (CV).

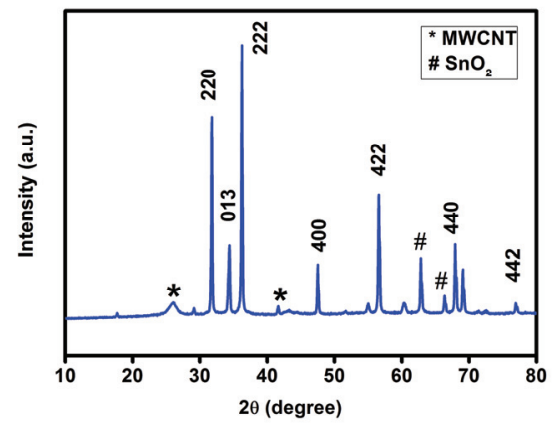

Figure 1: X-Ray Diffractogram of CNT-ZTO nanohybrid
Figure 1 shows two low intensity peaks at $26.1^{\circ}$ and $43.4^{\circ}$ corresponding to MWCNTs and two peaks at $62.7^{\circ}$ and $66.3^{\circ}$ due to tin oxide $\left(\mathrm{SnO}_{2}\right)$. All other peaks in the XRD plot correspond to ZTO, which were matched with JCPDS card no.11-0274.

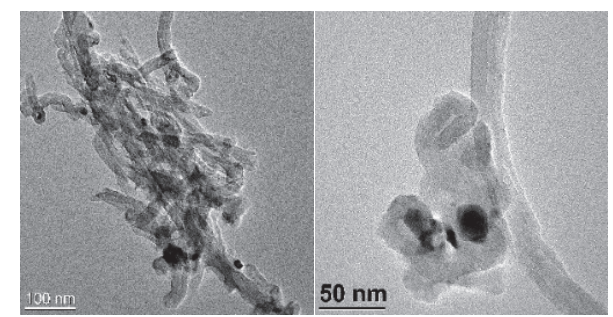

Figure 2: HRTEM images of synthesized CNT-ZTO nanohybrid

The images in Figure 2 show well resolved CNTs on which ZTO nanoparticles are attached. The calculated average particle size of ZTO falls within the range of 10-25 $\mathrm{nm}$.

In order to investigate the potential of the nanohybrids as electrode material for supercapacitor, the samples were characterised by cyclic voltammetry (CV) measurement. CV plots (not shown) recorded on these materials deposited on a glassy carbon working electrode, $\mathrm{Ag} / \mathrm{AgCl}$ as reference electrode and $\mathrm{Pt}$ as counter electrode revealed increasing values of current density for increasing scan rate when measured against applied potential. Calculations based on these plots suggest that specific capacitance values as high as $\sim 1250 \mathrm{~F} / \mathrm{g}$ may be achieved from the nanohybrid electrodes showing its potential use in supercapacitor. The results suggest that the superior conductive property combined with large surface area of CNT played a key role in improving the electron transport and in turn, excellent supercapacitive behaviour of the CNT- ZTO nanohybrid.

\section{References}

1. C. H. Fang, B. Y. Geng, J. Liu and F. M. Zhan, Chem.Commun. (2009) 2350-2352.

2. X. J. Zhu, L. M. Geng, F. Q. Zhang, Y. X. Liu and L. B. Cheng, J Power Sources 189 (2009) 828-831.

3. L. H. Huang, L. L. Jiang and M. D. Wei, Electrochem. Commun. 12 (2010) 319-322.

4. H.Pan,J.Li, and Y.Feng, Nanoscale Res Lett. 5 (2010) $654-668$ 